\title{
The association between risk of airflow limitation and serum uric acid measured at medical health check-ups
}

This article was published in the following Dove Press journal:

International Journal of COPD

19 April 2017

Number of times this article has been viewed

\author{
Atsuro Fukuhara' \\ Junpei Saito' \\ Suguru Sato' \\ Kazue Saito' \\ Naoko Fukuhara' \\ Yoshinori Tanino' \\ Xintao Wang' \\ Katsuaki Rinno² \\ Hitoshi Suzuki² \\ Mitsuru Munakata \\ 'Department of Pulmonary Medicine, \\ School of Medicine, Fukushima \\ Medical University, ${ }^{2}$ General \\ Examination Center, Fukushima \\ Preservation Service Association of \\ Health, Fukushima, Japan
}

\begin{abstract}
The prevalence of COPD and asthma is increasing all over the world; however, their morbidities are thought to be greatly underestimated because of unawareness of patients' conditions and respiratory symptoms. Spirometry is useful for the early detection of COPD and asthma with airflow limitation (AL), although it is not yet widely used for screening in epidemiological and primary care settings. A simple predictive marker used in combination with spirometry for AL is expected to be established. In medical health check-ups, serum uric acid (s-UA) is measured when screening for gout and has recently been suggested to have an association with several respiratory disorders, including asthma and COPD. However, whether s-UA influences the development of AL remains unclear. Therefore, the aims of this study were to examine the relationship between $\mathrm{AL}$ and s-UA and to investigate s-UA as a potential auxiliary marker for predicting AL risk in medical health check-ups. A total of 8,662 subjects aged $>40$ years were included. They were administered a simple questionnaire and assessed using pulmonary function tests, blood pressure (BP) measurements, and blood samplings. One hundred and fifty-six subjects (1.8\%) had AL, just $29 \%$ of whom had experienced respiratory symptoms. The subjects with AL had significantly higher s-UA levels compared with never-smoking subjects without AL. Forced expiratory volume in 1 second $\left(\mathrm{FEV}_{1}\right)$ \%predicted showed significant correlations with age, smoking index, body mass index (BMI), mean BP, white blood cells, hemoglobin A1c, s-UA, and high-density lipoprotein cholesterol. In multiple logistic regression analysis, s-UA, in addition to age, smoking index, respiratory symptoms, and BMI, was independently associated with AL. In conclusion, elevated s-UA levels, together with respiratory symptoms, high smoking index, and weight loss, may epidemiologically predict the development of AL risk.
\end{abstract}

Keywords: uric acid, airflow limitation, COPD, asthma, health check-up

\section{Introduction}

Asthma and COPD are major chronic airway diseases (CADs) that lead to airflow limitation (AL), with high and increasing prevalence all over the world. ${ }^{1,2}$ Based on the International Asthma Guidelines, 300 million people worldwide are estimated to have asthma, and the rates vary among countries with prevalence between $1 \%$ and $18 \% .{ }^{1}$ COPD is currently the fourth leading global cause of death and is likely to become the third cause by 2020. ${ }^{3}$ A meta-analysis of studies published between 1990 and 2004 revealed that the prevalence of COPD was estimated to be $9 \%-10 \%$ around the world. ${ }^{4}$ In Japan, the prevalence of asthma with AL and that of COPD in patients aged $>40$ years have been reported to be $\sim 2.0 \%$ and $8.9 \%$, respectively., $\mathrm{A}$ recent epidemiological investigation conducted by the Japanese Ministry of Health, Labour
Correspondence: Junpei Saito Department of Pulmonary Medicine, School of Medicine, Fukushima Medical University, I Hikarigaoka, Fukushima City, Fukushima Prefecture 960-I295, Japan

Tel +8 I 24547 I 360

Fax +8I 245489366

Email junpei@fmu.ac.jp 
and Welfare reported that $>9$ million and 5 million Japanese were estimated to have asthma and COPD, respectively. However, the number of actual reported cases of asthma and COPD is 1.2 million and 0.2 million, respectively. This discrepancy indicates that the reported prevalence of CADs may be the tip of the iceberg, and its morbidity has been greatly underestimated. The early detection of CADs is therefore crucial, before they become clinically apparent and advanced. However, patients with early-stage CADs may be either unaware of their condition or reluctant to consult their physician for respiratory symptoms. ${ }^{7}$ One of the best ways to detect CADs early on could be to screen for AL either in general practice or at medical health check-ups. However, only subjects suffering from respiratory symptoms are candidates for spirometry. Furthermore, spirometry is not yet sufficiently widespread within primary care settings or medical health check-ups. ${ }^{8}{ }^{8}$ It would be useful if there were a simple predictive marker used in combination with spirometry for AL in epidemiological settings.

Serum uric acid (s-UA) is the final product of purine degradation. ${ }^{10}$ In epidemiological settings, s-UA is frequently measured when screening for gout and when assessing renal dysfunction. Recently, s-UA has also been suggested to have an association with respiratory disorders such as stable COPD, ${ }^{11}$ asthma with acute exacerbation, COPD with acute exacerbation, ${ }^{12,13}$ obstructive sleep apnea, ${ }^{14}$ pulmonary hypertension, ${ }^{15}$ and chronic respiratory failure. ${ }^{16,17}$ From a screening point of view, early identification of subjects with possible risk of AL may enable appropriate stratification of prevention and treatment. However, an association between s-UA and the risk of AL has been less investigated in an epidemiological setting.

The aims of this study were to examine the relationship between AL and s-UA and to investigate s-UA as a potential auxiliary marker for predicting AL risk in a medical health check-up.

\section{Methods}

\section{Study population and design}

This is an epidemiological study based on an annual community health check-up at the Fukushima Preservation Service Association of Health in Fukushima Prefecture, Japan. A total of 8,783 subjects aged $>40$ years were registered, of whom 121 subjects were excluded because of incomplete data of pulmonary function and blood testing. After exclusion, 8,662 subjects (5,191 men and 3,471 women) were entered into the data analysis. All subjects answered a simple self-reported questionnaire that included questions on respiratory symptoms (coughing, sputum, and dyspnea), smoking status, and medical histories. In addition, pulmonary function tests, blood pressure (BP) measurements, ${ }^{18}$ and blood samplings, including peripheral blood count, blood glucose levels, hemoglobin A1c (HbA1c), serum levels of total protein (TP), creatinine (Crea), UA, total cholesterol (TC), and high-density lipoprotein cholesterol (HDL-C), were conducted as routine screening tests for lifestyle-related disorders. This study was approved by the local ethics committee of Fukushima Medical University and Fukushima Preservation Service Association of Health. All participants provided written informed consent.

\section{Pulmonary function tests}

Spirometry was performed (Autospiro system- $7^{\circledR}$; Minato Medical Science, Osaka, Japan). A bronchodilator was not administered prior to measurements because of ethical restrictions. Two or three repeated measurements were taken by well-experienced respiratory technicians according to the American Thoracic Society/European Respiratory Society guidelines, ${ }^{19}$ and the highest technically acceptable measurements reproducible to within $150 \mathrm{~mL}$ were retained. If the differences between two or three measurements were $>150 \mathrm{~mL}$, additional trials were attempted, with up to eight measurements.

\section{Classification of subjects}

All data were assessed retrospectively. AL was defined as forced expiratory volume in 1 second/forced vital capacity $\left(\mathrm{FEV}_{1} / \mathrm{FVC}\right)<0.7 .^{19}$

As for the definition of smoking status, subjects were categorized as current smokers, former smokers, and neversmokers. Never-smokers were subjects who denied past and current smoking. Former smokers were those who previously smoked but denied current smoking. Current smokers were those who reported that they currently smoked at least one cigarette a day. The Brinkman index, defined as multiplying the number of cigarettes smoked per day by smoking years, was used as a smoking index to estimate how the subjects were exposed to smoking.

\section{Statistical analysis}

Statistical analyses were performed using SPSS for Windows version 21.0 software (SPSS Inc, Chicago, IL, USA). Data are shown as mean \pm standard deviation. All data were non-normally distributed, which was confirmed using the Kolmogorov-Smirnov test. Comparisons of continuous variables between subjects with and without AL were made 
using the Mann-Whitney $U$ test. A chi-square test was used for the comparison of categorical data. Multiple comparisons of s-UA levels were performed by the Kruskal-Wallis test. The correlations between $\mathrm{FEV}_{1}$ \% predicted and parameters that may be related to AL were evaluated by Spearman's rank correlation coefficient analysis. Finally, multiple logistic regression analysis was conducted to determine the independent associations of AL with parameters with $P$-value $<0.1$ obtained from comparisons between subjects with AL and those without AL. A two-tailed $P$-value of $<0.05$ was considered statistically significant.

\section{Results}

\section{Characteristics of subjects}

Table 1 shows the characteristics of the subjects divided into two groups according to AL. There were 156 subjects (1.8\%) with AL, only $29 \%$ (45 of 156 ) of whom exhibited respiratory symptoms (coughing, sputum, or dyspnea). The number of current and former smokers in the AL group was 65 (41.4\%) and 49 (30.8\%), respectively, which were significantly higher proportions compared to the current and former smokers without AL $(P<0.0001)$. In addition, the proportions of male gender, age, smoking index, and white blood cell (WBC) count were significantly higher in the subjects with AL than in those without $(P<0.05)$. On the other hand, body mass index (BMI), TP, \% vital capacity (\%VC), $\mathrm{FEV}_{1} \%$ predicted, and $\mathrm{FEV}_{1} / \mathrm{FVC}$ were all significantly lower in the subjects with
AL than in those without $(P<0.05)$. In addition, HbA1c and $\mathrm{TC}$ in the AL subjects showed higher and lower trends compared to those in the subjects without AL, respectively. BP, Crea, and HDL-C did not differ between the two groups.

\section{s-UA levels in the subjects with and without $A L$}

Subjects with AL had higher s-UA levels compared to never-smoking subjects without AL (5.5 \pm 1.5 vs $4.8 \pm 1.3$, $P<0.001)$. In addition, s-UA levels in former smoking and current smoking subjects without AL (5.9 \pm 1.4 and 5.7 \pm 1.4 , respectively) were also higher than those in never-smoking subjects without AL $(P<0.001)$ (Figure 1).

\section{Relationships between FEV, \%predicted and relevant parameters}

Table 2 shows the correlations between $\mathrm{FEV}_{1}$ \% predicted and parameters measured during medical check-ups. FEV $\%$ predicted was positively associated with age $(r=0.13$, $P<0.001)$ and HDL-C $(r=0.11, P<0.001)$ and was negatively correlated with smoking index $(r=-0.14, P<0.001)$, BMI $(r=-0.05, P<0.001)$, mean BP $(r=-0.03, P=0.002)$, WBC count $(r=-0.12, P<0.001)$, HbA1c $(r=-0.06, P<0.001)$, and s-UA ( $r=-0.06, P<0.001)$ (Figure 2$)$.

\section{Risk factors that influence $A L$}

Multiple logistic regression analysis to determine the relevant parameters for predicting AL risk showed that increased

Table I Comparison of individual parameters between subjects with and without $\mathrm{AL}$

\begin{tabular}{|c|c|c|c|c|}
\hline & Total $(n=8,662)$ & AL $(+)(n=156)$ & AL $(-)(n=8,506)$ & $P$-value \\
\hline Gender (male/female) & $5,|9| / 3,47 \mid$ & $117 / 39$ & $5,074 / 3,432$ & $<0.001$ \\
\hline Age, years & $52.5(7.6)$ & $58.4(8.1)$ & $52.4(7.6)$ & $<0.001$ \\
\hline Smoking index & $260(36 I)$ & $555(50 \mathrm{I})$ & $254(356)$ & $<0.001$ \\
\hline Smoking status (C/F/N) & $2,493 / 1,896 / 4,273$ & $65 / 49 / 42$ & $2,428 / I, 847 / 4,23 \mid$ & $<0.001$ \\
\hline Respiratory symptoms $( \pm)$ & I, I79/7,483 & $45 / 111$ & $\mathrm{I}, 134 / 7,372$ & $<0.001$ \\
\hline Height, cm & $163.0(8.8)$ & I 63.7 (7.9) & $162.9(8.8)$ & 0.2 \\
\hline Body weight, kg & $62.8(11.2)$ & $60.9(11.8)$ & $62.8(11.1)$ & 0.02 \\
\hline $\mathrm{BMI}, \mathrm{kg} / \mathrm{m}^{2}$ & $23.6(3.1)$ & $22.6(3.4)$ & $23.6(3.1)$ & $<0.001$ \\
\hline$\% \mathrm{VC}, \%$ & $101.6(14.2)$ & $92.0(20.8)$ & $101.7(14.0)$ & $<0.001$ \\
\hline $\mathrm{FEV}_{1} / \mathrm{FVC}, \%$ & $84.0(6.2)$ & $63.9(6.1)$ & $84.3(5.5)$ & $<0.001$ \\
\hline Mean BP, mmHg & $91.9(11.7)$ & $91.9(12.0)$ & $91.9(11.7)$ & 0.9 \\
\hline $\mathrm{WBC}, / \mu \mathrm{L}$ & $5,635(1,631)$ & $6,090(1,922)$ & $5,627(1,624)$ & 0.003 \\
\hline $\mathrm{TP}, \mathrm{g} / \mathrm{dL}$ & $7.2(0.4)$ & $7.1(0.4)$ & $7.2(0.4)$ & 0.01 \\
\hline Crea, mg/dL & $0.8(0.3)$ & $0.8(0.2)$ & $0.8(0.3)$ & 0.8 \\
\hline $\mathrm{HbAlc}, \%$ & $5.3(0.8)$ & $5.4(0.8)$ & $5.2(0.8)$ & 0.05 \\
\hline $\mathrm{TC}, \mathrm{mg} / \mathrm{dL}$ & $202.7(33.1)$ & $198.0(33.2)$ & $202.8(33.0)$ & 0.06 \\
\hline $\mathrm{HDL}-\mathrm{C}, \mathrm{mg} / \mathrm{dL}$ & $56.6(14.0)$ & $55.6(13.6)$ & $56.6(14.0)$ & 0.3 \\
\hline
\end{tabular}

Note: Data are presented as mean (SD) unless otherwise indicated.

Abbreviations: AL, airflow limitation; C, current smoker; F, former smoker; N, never-smoker; BMI, body mass index; VC, vital capacity; FEV ${ }_{1}$, forced expiratory volume in I second; FVC, forced vital capacity; BP, blood pressure; WBC, white blood cell; TP, total protein; Crea, creatinine; HbAIc, hemoglobin AI c; TC, total cholesterol; HDL-C, high-density lipoprotein cholesterol; SD, standard deviation. 


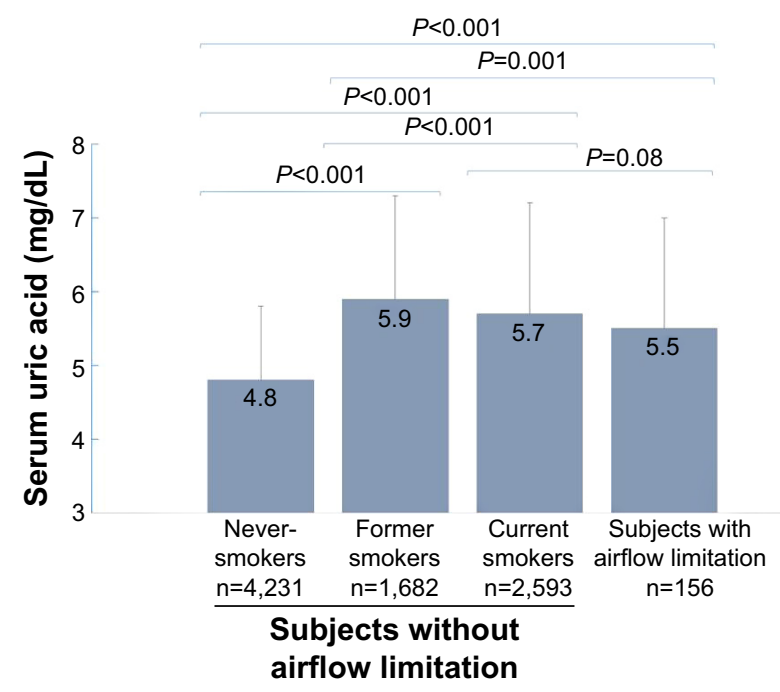

Figure I s-UA levels in subjects with and without airflow limitation.

Notes: Former and current smokers without AL had higher s-UA levels compared to never-smokers without AL. Additionally, subjects with AL also had higher s-UA levels than never-smokers without $\mathrm{AL}$.

Abbreviations: s-UA, serum uric acid; $\mathrm{AL}$, airflow limitation.

s-UA (odds ratio [OR], 1.158; 95\% confidence interval [95\% CI], 1.003-1.337; $P=0.04)$ in addition to age, high smoking index, positive respiratory symptoms, and low BMI (OR, 1.093, 1.001, 2.017, and 0.855, respectively; $P<0.001$ ) could be an independent risk factor of AL (Table 3 ).

\section{Discussion}

In this population-based epidemiological study, there were 156 subjects (1.8\%) with AL. However, only 29\% (45 of 156 subjects) of those experienced respiratory symptoms. In addition, the subjects with AL had significantly higher s-UA levels compared to never-smoking subjects without AL.

Table 2 Correlation analysis between FEV, \%predicted and parameters measured at medical health check-ups

\begin{tabular}{lll}
\hline & $\boldsymbol{r}$ & $\boldsymbol{P}$-value \\
\hline Age & 0.13 & $<0.001$ \\
Smoking index & -0.14 & $<0.001$ \\
BMI & -0.05 & $<0.001$ \\
Mean BP & -0.03 & 0.002 \\
WBC & -0.12 & $<0.001$ \\
TP & -0.01 & 0.3 \\
Crea & 0.003 & 0.7 \\
HbAIc & -0.06 & $<0.001$ \\
s-UA & -0.06 & $<0.001$ \\
TC & 0.006 & 0.5 \\
HDL-C & 0.11 & $<0.001$ \\
\hline
\end{tabular}

Note: Data are presented as $r$ value.

Abbreviations: $\mathrm{FEV}_{\text {, }}$, forced expiratory volume in I second; $\mathrm{BMI}$, body mass index; $\mathrm{BP}$, blood pressure; WBC, white blood cell; TP, total protein; Crea, creatinine; HbAlc, hemoglobin Alc; s-UA, serum uric acid; TC, total cholesterol; HDL-C, high-density lipoprotein cholesterol.

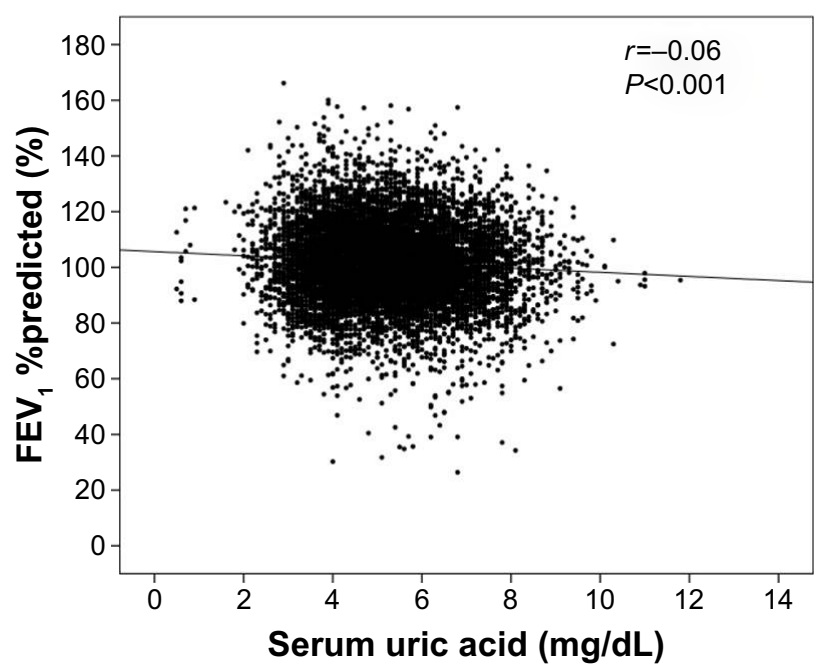

Figure 2 Relationship between FEV, \%predicted and s-UA.

Note: $A$ weak but significant correlation was observed between FEV, \%predicted and s-UA.

Abbreviations: $\mathrm{FEV}_{\text {, }}$, forced expiratory volume in I second; s-UA, serum uric acid.

Moreover, the degree of AL expressed by $\mathrm{FEV}_{1}$ \%predicted showed weak but significant correlations with age, smoking index, BMI, mean BP, WBC, HbAlc, s-UA, and HDL-C. Finally, multiple logistic regression analysis revealed that s-UA levels, as well as age, smoking index, respiratory symptoms, and BMI, were independently associated with AL.

In this study, $71 \%$ of subjects with $\mathrm{AL}$ were asymptomatic at examination, which is slightly higher compared to other epidemiological studies, where $40 \%-60 \%$ of the subjects with AL were free of respiratory symptoms. ${ }^{6,20,21}$ A possible reason for this could be that the number of subjects with severe AL in this study was relatively small compared to the other studies. In any case, from an early diagnostic point of view, respiratory symptoms do not always predict AL, ${ }^{22}$

Table 3 Multiple logistic regression analysis (association with $\mathrm{AL}$ )

\begin{tabular}{|c|c|c|c|}
\hline & OR & $95 \% \mathrm{Cl}$ & P-value \\
\hline Gender & 0.885 & $0.506-1.549$ & 0.6 \\
\hline Age & 1.093 & $1.071-1.116$ & $<0.001$ \\
\hline Smoking index & 1.001 & $1.001-1.001$ & $<0.001$ \\
\hline Smoking status & 0.940 & $0.705-1.255$ & 0.6 \\
\hline Respiratory symptoms & 2.017 & $|.392-2.92|$ & $<0.001$ \\
\hline BMI & 0.855 & $0.804-0.909$ & $<0.001$ \\
\hline WBC & 1.088 & $0.988-1.197$ & 0.08 \\
\hline TP & 0.836 & $0.538-1.299$ & 0.4 \\
\hline Crea & 0.323 & $0.097-1.080$ & 0.06 \\
\hline $\mathrm{HbAlc}$ & 1.036 & $0.859-1.251$ & 0.7 \\
\hline s-UA & 1.158 & $1.003-1.337$ & 0.04 \\
\hline $\mathrm{TC}$ & 0.998 & $0.993-1.003$ & 0.4 \\
\hline
\end{tabular}

Note: Data are presented as adjusted OR and $95 \% \mathrm{Cl}$.

Abbreviations: $\mathrm{AL}$, airflow limitation; $\mathrm{OR}$, odds ratio; $\mathrm{Cl}$, confidence interval; BMI, body mass index; WBC, white blood cell; TP, total protein; Crea, creatinine; HbAIc, hemoglobin AIc; s-UA, serum uric acid; TC, total cholesterol. 
and spirometry is essential for early detection of AL. ${ }^{6,20,21,23}$ Although spirometry has become more readily available in recent years, it is not yet used widespread for the diagnosis of CADs in both the epidemiological and primary care settings. ${ }^{8,9}$ Further campaigns are needed to promote the expansion of the use of spirometry.

COPD, one of the major causes of CADs, is characterized by chronic AL, which is associated with chronic airway inflammation. Recently, COPD has also been recognized to be a part of systemic inflammation, leading to considerable comorbidities such as cardiovascular diseases, cerebrovascular diseases, lung cancer, diabetes, hypertension, hyperlipidemia, chronic renal failure, osteoporosis, and muscular weakness. ${ }^{24}$ Nevertheless, a diagnosis of COPD is made according to just the patient's symptoms and spirometric measurements of $\mathrm{FEV}_{1} / \mathrm{FVC}<0.7$. Markers of either airway or systemic inflammation are not included. Thus, systemic biomarkers associated with airway inflammation and AL would be helpful for the early detection of COPD. In previous studies, several potential and clinically relevant blood biomarkers, such as high sensitive $\mathrm{C}$-reactive protein, fibrinogen, interleukin 6 , surfactant protein D, and Clara cell secretory protein-16, have been identified to discriminate COPD from healthy controls. ${ }^{25,26}$ However, they were usually examined on certain occasions only, including exacerbation, hospitalization, and clinical research settings (not routinely performed at health check-ups). It would be better to identify a biomarker that is widely available in epidemiological settings.

Interestingly, s-UA levels in subjects with AL in this study were higher than those in the never-smoking subjects without $\mathrm{AL}$ and significantly correlated with $\mathrm{FEV}_{1}$ $\%$ predicted. Furthermore, increased s-UA was one of the independent parameters associated with AL by multiple logistic regression analysis. Recently, s-UA has been suggested to have an association with hypertension, metabolic syndrome, and cardiovascular diseases. ${ }^{27}$ However, the relationship between s-UA and respiratory disorders remains unclear. Several studies have shown that s-UA is increased in hypoxic conditions, including in patients with stable COPD ${ }^{11}$ asthma with exacerbation, COPD with acute exacerbation, ${ }^{12,13}$ obstructive sleep apnea, ${ }^{14}$ pulmonary hypertension, ${ }^{15}$ and chronic respiratory failure. ${ }^{16,17}$ In a large population-based epidemiological study, Aida et $\mathrm{al}^{28}$ reported that s-UA levels were negatively correlated with the presence of AL. All results from the aforementioned studies, including this study, provide evidence that s-UA could be considered as one of the candidate biomarkers for predicting future risk of AL.
However, other studies have reported conflicting results. In a large population-based cohort study, Horsfall et $\mathrm{al}^{29}$ revealed that low levels of s-UA were associated with higher rates of COPD and lung cancer. Similarly, Nicks et $\mathrm{al}^{30}$ reported reduced s-UA levels in patients with severe COPD. This contradiction may be due to the dual function of s-UA, namely, having either a pro-inflammatory or an anti-inflammatory effect under different conditions. At present, the pathophysiological role of s-UA in the systemic circulation remains unknown. UA is the final product of purine degradation, ${ }^{10}$ and its production is catalyzed by xanthine oxidase. Some studies have suggested that increased xanthine oxidase activity under hypoxic conditions could promote the overproduction of UA, which may lead to tissue and organ damage. ${ }^{10,31,32}$ Conversely, other previous studies have reported that s-UA in healthy subjects alleviated oxidative stress during physical training and that s-UA could restore endothelial function in healthy smoking subjects and patients with type 1 diabetes, ${ }^{33,34}$ suggesting that s-UA may have an antioxidant effect. The fact that increased s-UA was independently associated with AL in our study could indicate a possible role of s-UA in worsening airway conditions. Further studies are necessary to clarify the mechanisms of $\mathrm{s}-\mathrm{UA}$ in the systemic circulation.

This study has several limitations. First, the prevalence of AL (1.8\%) was lower compared to other studies. ${ }^{4,6,35}$ The reason for this could be that only $17 \%$ of our study population was $>60$ years old, which is lower than other studies. This may have influenced the overall difference in parameters relevant to CADs. Second, we could not completely rule out all possible factors that may influence s-UA levels, including chronic kidney disorders, genetic disorders of purine metabolism, cardiovascular diseases, food intake, metabolic syndrome, and alcohol consumption. ${ }^{27,36,37}$ However, even after adjusting for possible and major confounding factors (age, gender, BMI, and smoking index), logistic regression analysis showed that s-UA levels continued to be an independent predictor of AL risk. We believe that the influence of bias from unknown confounding factors was small and did not significantly affect our results. Third, causes of AL could not be identified in this study because any specific examinations for definite diagnoses have not been performed However, the main purpose of this study was to evaluate the utility of s-UA as a screening marker for predicting AL risk epidemiologically. Further follow-up studies would clarify the relationship between s-UA and specific disorders causing AL. Fourth, pre-bronchodilator values were used to assess AL, which can overestimate the prevalence of AL. 
However, we are not ethically allowed to use bronchodilators in the epidemiological screening unlike many previous epidemiological studies on AL detection. ${ }^{38-40}$ In addition, the purpose of this study is to evaluate the relationship between s-UA and AL but not to estimate the prevalence of AL. Thus, we believe that the use of pre-bronchodilator values rather than post-bronchodilator values would not significantly alter the main result that s-UA can independently predict the AL risk since there is a linear significant correlation between $\mathrm{FEV}_{1}$ and s-UA.

\section{Conclusion}

We have shown that s-UA is independently associated with AL. Elevated s-UA, together with respiratory symptoms, high smoking index, and weight loss, may provide better information to predict AL risk in an epidemiological setting. A further longitudinal follow-up study is needed as a next step to estimate the relationship between s-UA and the annual progression of AL.

\section{Acknowledgments}

We would like to thank Dr Yayoi Inokoshi, Dr Taeko Ishii, Dr Takashi Ishida, and Dr Hideo Muraoka for their statistical advice and constructive comments. We would also like to thank Mr Pete McCann for his linguistic assistance in proofreading the manuscript.

\section{Disclosure}

The authors report no conflicts of interest in this work.

\section{References}

1. Global Initiative for Asthma [Homepage on the Internet]. Global Strategy for Asthma Management and Prevention. Available from: www. ginasthma.org. Accessed July 25, 2016.

2. The Global Initiative for Chronic Obstructive Lung Disease [Homepage on the Internet]. Global Strategy for Diagnosis, Management, and Prevention of COPD-2016. Available from: http://goldcopd.org. Accessed July 25, 2016.

3. Murray CJ, Lopez AD. Mortality by cause for eight regions of the world: global burden of disease study. Lancet. 1997;349(9061):1269-1276.

4. Halbert RJ, Natoli JL, Gano A, Badamgarav E, Buist AS, Mannino DM. Global burden ofCOPD: systematic review and meta-analysis. Eur Respir J. 2006;28(3):523-532.

5. Matsumoto K, Seki N, Fukuyama S, et al. Prevalence of asthma with airflow limitation, COPD, and COPD with variable airflow limitation in older subjects in a general Japanese population: the Hisayama Study. Respir Investig. 2015;53(1):22-29.

6. Fukuchi Y, Nishimura M, Ichinose M, et al. COPD in Japan: the Nippon COPD Epidemiology study. Respirology. 2004;9(4):458-465.

7. van den Boom G, van Schayck CP, van Mollen MP, et al. Active detection of chronic obstructive pulmonary disease and asthma in the general population. Results and economic consequences of the DIMCA program. Am J Respir Crit Care Med. 1998;158(6):1730-1738.

8. Arne M, Lisspers K, Stallberg B, et al. How often is diagnosis of COPD confirmed with spirometry? Respir Med. 2010;104(4):550-556.
9. Han MK, Kim MG, Mardon R, et al. Spirometry utilization for COPD: how do we measure up? Chest. 2007;132(2):403-409.

10. Grassi D, Ferri L, Desideri G, et al. Chronic hyperuricemia, uric acid deposit and cardiovascular risk. Curr Pharm Des. 2013;19(13): 2432-2438

11. Garcia-Pachon E, Padilla-Navas I, Shum C. Serum uric acid to creatinine ratio in patients with chronic obstructive pulmonary disease. Lung. 2007;185(1):21-24.

12. Li L, Wan C, Wen F. An unexpected role for serum uric acid as a biomarker for severity of asthma exacerbation. Asian Pac J Allergy Immunol. 2014;32(1):93-99.

13. Bartziokas K, Papaioannou AI, Loukides S, et al. Serum uric acid as a predictor of mortality and future exacerbations of COPD. Eur Respir J. 2014;43(1):43-53.

14. Saito H, Nishimura M, Shibuya E, et al. Tissue hypoxia in sleep apnea syndrome assessed by uric acid and adenosine. Chest. 2002;122(5): 1686-1694.

15. Voelkel MA, Wynne KM, Badesch DB, Groves BM, Voelkel NF. Hyperuricemia in severe pulmonary hypertension. Chest. 2000;117(1): $19-24$.

16. Sato N, Kurashima K, Ubukata M, et al. [Prognostic significance of serum uric acid in patients with chronic obstructive pulmonary disease receiving home oxygen therapy]. Nihon Kokyuki Gakkai Zasshi. 2003;41(2):74-80. Japanese.

17. Kadowaki T, Hamada H, Yokoyama A, et al. Significance of serum uric acid in patients with chronic respiratory failure treated with non-invasive positive pressure ventilation. Intern Med. 2007;46(11):691-697.

18. Zheng L, Sun Z, Li J, et al. Pulse pressure and mean arterial pressure in relation to ischemic stroke among patients with uncontrolled hypertension in rural areas of China. Stroke. 2008;39(7):1932-1937.

19. Miller MR, Hankinson J, Brusasco V, et al. Standardisation of spirometry. Eur Respir J. 2005;26(2):319-338.

20. Chang JH, Lee JH, Kim MK, et al. Determinants of respiratory symptom development in patients with chronic airflow obstruction. Respir Med. 2006;100(12):2170-2176.

21. Bridevaux PO, Probst-Hensch NM, Schindler C, et al. Prevalence of airflow obstruction in smokers and never-smokers in Switzerland. Eur Respir J. 2010;36(6):1259-1269.

22. Renwick DS, Connolly MJ. Do respiratory symptoms predict chronic airflow obstruction and bronchial hyperresponsiveness in older adults? J Gerontol A Biol Sci Med Sci. 1999;54(3):M136-M139.

23. Buffels J, Degryse J, Heyrman J, Decramer M, Study D. Office spirometry significantly improves early detection of COPD in general practice: the DIDASCO Study. Chest. 2004;125(4):1394-1399.

24. Divo M, Cote C, de Torres JP, et al. Comorbidities and risk of mortality in patients with chronic obstructive pulmonary disease. Am J Respir Crit Care Med. 2012;186(2):155-161.

25. Dickens JA, Miller BE, Edwards LD, et al. COPD association and repeatability of blood biomarkers in the ECLIPSE cohort. Respir Res. 2011;12:146.

26. Vestbo J, Agusti A, Wouters EF, et al. Should we view chronic obstructive pulmonary disease differently after ECLIPSE? A clinical perspective from the study team. Am J Respir Crit Care Med. 2014; 189(9):1022-1030.

27. Feig DI, Kang DH, Johnson RJ. Uric acid and cardiovascular risk. N Engl J Med. 2008;359(17):1811-1821.

28. Aida Y, Shibata Y, Osaka D, et al. The relationship between serum uric acid and spirometric values in participants in a health check: the Takahata study. Int J Med Sci. 2011;8(6):470-478.

29. Horsfall LJ, Nazareth I, Petersen I. Serum uric acid and the risk of respiratory disease: a population-based cohort study. Thorax. 2014; 69(11):1021-1026.

30. Nicks ME, O’Brien MM, Bowler RP. Plasma antioxidants are associated with impaired lung function and COPD exacerbations in smokers. COPD. 2011;8(4):264-269.

31. Elsayed NM, Nakashima JM, Postlethwait EM. Measurement of uric acid as a marker of oxygen tension in the lung. Arch Biochem Biophys. 1993;302(1):228-232. 
32. Puig JG, Ruilope LM. Uric acid as a cardiovascular risk factor in arterial hypertension. J Hypertens. 1999;17(7):869-872.

33. Waring WS, McKnight JA, Webb DJ, Maxwell SR. Uric acid restores endothelial function in patients with type 1 diabetes and regular smokers. Diabetes. 2006;55(11):3127-3132.

34. Waring WS, Convery A, Mishra V, Shenkin A, Webb DJ, Maxwell SR. Uric acid reduces exercise-induced oxidative stress in healthy adults. Clin Sci (Lond). 2003;105(4):425-430.

35. Rennard S, Decramer M, Calverley PM, et al. Impact of COPD in North America and Europe in 2000: subjects' perspective of Confronting COPD International Survey. Eur Respir J. 2002;20(4):799-805.

36. Feig DI. Uric acid: a novel mediator and marker of risk in chronic kidney disease? Curr Opin Nephrol Hypertens. 2009;18(6):526-530.
37. Stiburkova B, Pavlikova M, Sokolova J, Kozich V. Metabolic syndrome, alcohol consumption and genetic factors are associated with serum uric acid concentration. PLoS One. 2014;9(5):e97646.

38. Roche N, Dalmay F, Perez T, et al. FEV1/FVC and FEV1 for the assessment of chronic airflow obstruction in prevalence studies: do prediction equations need revision? Respir Med. 2008;102(11):1568-1574.

39. de Marco R, Accordini S, Cerveri I, et al. An international survey of chronic obstructive pulmonary disease in young adults according to GOLD stages. Thorax. 2004;59(2):120-125.

40. de Marco R, Accordini S, Cerveri I, et al. Incidence of chronic obstructive pulmonary disease in a cohort of young adults according to the presence of chronic cough and phlegm. Am J Respir Crit Care Med. 2007; 175(1):32-39.

\section{Publish your work in this journal}

The International Journal of COPD is an international, peer-reviewed journal of therapeutics and pharmacology focusing on concise rapid reporting of clinical studies and reviews in COPD. Special focus is given to the pathophysiological processes underlying the disease, intervention programs, patient focused education, and self management protocols.

\section{Dovepress}

This journal is indexed on PubMed Central, MedLine and CAS. The manuscript management system is completely online and includes a very quick and fair peer-review system, which is all easy to use. Visit http://www.dovepress.com/testimonials.php to read real quotes from published authors.

Submit your manuscript here: http://www.dovepress.com/international-journal-of-chronic-obstructive-pulmonary-disease-journal 